\title{
La pensée mariale de Mgr de Saint-Vallier
}

\section{Hector Bibeau}

Volume 33, 1966

URI : https://id.erudit.org/iderudit/1007317ar

DOI : https://doi.org/10.7202/1007317ar

Aller au sommaire du numéro

Éditeur(s)

Les Éditions Historia Ecclesiæ Catholicæ Canadensis Inc.

ISSN

0318-6172 (imprimé)

1927-7067 (numérique)

Découvrir la revue

Citer cet article

Bibeau, H. (1966). La pensée mariale de Mgr de Saint-Vallier. Sessions d'étude -

Société canadienne d'histoire de l'Église catholique, 33, 17-23.

https://doi.org/10.7202/1007317ar

Tous droits réservés @ Les Éditions Historia Ecclesiæ Catholicæ Canadensis Inc., 1967
Ce document est protégé par la loi sur le droit d'auteur. L'utilisation des services d'Érudit (y compris la reproduction) est assujettie à sa politique d'utilisation que vous pouvez consulter en ligne.

https://apropos.erudit.org/fr/usagers/politique-dutilisation/ 


\section{La pensée mariale de Mgr de Saint-Vallier}

En guise de préambule, je vous dirai simplement que mon propos se situe au point de confluence d'une donnée historique indiscutable et d'une hypothèse de travail qui me paraît valable. Cette donnée, c'est l'existence d'un courant de piété et de vie mariales au sein de la communauté canadienne à la fin du XVIle siècle. L'hypothèse, en résumé, voudrait que $\mathrm{Mgr}$ de Saint.Vallier constitue - vu la nature et la portée de son oeuvre pastorale - un relais important dans l'histoire du sentiment religieux de la Nouvelle-France.

On doit en effet tenir pour acquis que la piété populaire a pris, dès les origines de la colonie, l'un de ses plus constants appuis sur la dévotion à Marie. Il suffit de rappeler: l'action décisive des missionnaires Jésuites, initiateurs et propagateurs du Vœu de 1635; l'apport non moins assuré, encore que mal défini, de quelques ouvriers illustres par ailleurs - de l'Eglise canadienne, tels que Mgr de Laval, Catherine de Saint-Augustin, Marguerite Bourgeois et plusieurs autres; la vitalité des confréries mariales; la mise sous vocable marial de nombre d'églises et de chapelles votives. Ce ne sont là que des chefs de preuves, qu'un congrès antérieur de cette Société $a$, du reste, déjà mis en valeur ${ }^{1}$; ils font voir qu'il ne s'agit pas seulement d'explosions de ferveur momentanées mais d'une tradition mariale largement répandue, bien vivante, et qu'à l'arrivée de Mgr de Saint-Vallier (1688), NotreDame fait partie des attentions spirituelles et de la vie de la jeune Eglise canadienne.

D'autre part, il y a lieu de reconsidérer avec plus d'attention le rôle du deuxième évêque de Québec. N'a-t-il pas été, lui aussi, au sens plein du mot, l'un des fondateurs de l'Eglise canadienne? Le titre, j'en conviens, appartient plutôt à ces pionniers qui ont planté l'Eglise en terre canadienne. Mais est-ce trop présumer que de ranger avec eux l'artisan qui a transformé une Eglise jusque-là missionnaire en Eglise établie, en lui donnant des structures administratives définitives? Mgr de Saint-Vallier a posé les cadres d'une réforme analogue à celle entreprise par le roi - sur le plan politique - en 1663; de ce point de vue, il représente sûrement une étape. Mais il n'a pas que réorganisé l'Eglise canadienne; il fut aussi, pendant quarante-deux ans, en quelque sorte son magister spiritus par les quelque quatre-vingts mandements et lettres pastorales qu'il a écrits, les quatre synodes qu'il a dirigés et surtout par les deux auvres maîtresses qu'il a laissées et dont on n'a pas encore fini de prospecter toute l'influence ${ }^{2}$ : un Catéchisme paru

1 SCHEC, 2le congrès: rapport 1953-54.

2 Le Père Fernand PorTer, o.f.m., dans son Institution Catéchistique au Canada - Deux siècles de formation religieuse, 1633-1833 - présente une excellente étude du Catéchisme de Mgr de Saint-Vallier. Rien de comparable n'a été fait pour le Rituel. 
en 1702, qui sera pratiquement le seul manuel d'instruction et de formation religieuses pendant un demi-siècle et un Rituel, véritable compendium de la vie liturgique et sacramentaire - terminé en 1703 ne sera définitivement remplacé qu'en $1864^{3}$. Pareille abondance témoigne, entre autres choses, d'un zèle militant. Pareille survie prolonge la portée du message bien au-delà de la vie de son auteur.

Les écrits de Mgr de Saint-Vallier offrent donc un champ d'intérêt indéniable. Même si, à cause de l'état actuel de l'accès aux sources, on ne peut que très ditticilement départager ce qui lui appartient en propre, il peut être utile d'isoler l'un ou l'autre des grands thèmes de son message pour en mieux marquer les traits dominants.

Le thème marial dont il sera ici question n'est certes pas quantitativement le plus considérable. Il reste tout de même important vu la tradition que nous venons de signaler; il offre en outre ce singulier intérêt - que je voudrais partager avec vous - d'être un domaine d'investigation encore inexploré.

Nous essaierons de dire, ici, ce qui caractérise la pensée mariale de Mgr de Saint-Vallier. Encore est-il opportun cependant de dire, au préalable, où et comment elle se présente pour ne pas fausser les perspectives.

I - La pensée d'un praticien : circonstancielle, parcellaire.

Quel que soit l'angle sous lequel on examine les écrits de Mgr de Saint-Vallier, - la relation de 1688 exceptée, qui est une épître particulière, de propagande - on retrouve partout la même préoccupation foncière du pasteur dominé par le souci d'organiser, voire d'uniformiser la pratique religieuse dans son diocèse, de noyauter l'agir quotidien des fidèles commis à sa garde et le zèle de leurs pasteurs par ces deux pôles indispensables, selon lui, à une action durable : la famille et la paroisse. Il n'est pas le théoricien qui bat sa pensée à froid pour construire des arguments : il décide, recommande, fulmine ou soutient, bref prend position sous la pression des événements, des besoins ou des obstacles qu'il rencontre; Mgr de Saint-Vallier est un praticien. C'est dire jusqu'à quel point le débit, même l'expression, de sa pensée sont conditionnés, influencés par les circonstances immédiates. Voilà dans quelle perspective d'ensemble il faut d'abord situer sa pensée mariale.

Cette pensée n'a pas partout la même intensité ni la même allure selon qu'elle s'adresse aux curés ou aux gens du peuple. C'est une première distinction à retenir. Ajoutée au fait que l'évêque écrit toujours sous le feu de l'action, elle aide à comprendre que le contenu marial de sa pensée soit chargé de beaucoup de répétitions. Il importe davantage - je le dis au risque de répéter un lieu commun - de considérer la nature particulière ou la finalité propre de chacun des exposés; c'est

3 A. Gosseurn, L'Eglise du Canada, I : 232. Voir également Mandements / ... / des Evêques de Québec, III: 343; IV: 651. 
sur elles que se fondent principalement leurs ressemblances et leurs différences. On peut, de la sorte, ranger les écrits de Mgr de Saint-Vallier sous trois grandes rubriques : écrits didactiques, papiers officiels, papiers personnels.

Les écrits proprement didactiques, au nombre desquels il faut inscrire d'emblée le Catéchisme et le Rituel, sont les œuvres essentiellement doctrinales et magistrales qui, de soi, laissent peu de place à la spontanéité de l'inspiration, aux tendances personnelles et à la tradition locale. La pensée y est méthodique et (pour cause!) toujours prudente. C'est ici cependant, dans ces œuvres à caractère préceptif, que la pensée mariale de l'évêque prend son développement le plus extensif et un tour pédagogique vraiment intéressant. Hormis quelques jetées, souvent remarquables, distribuées ça et là à l'occasion d'un commentaire sur le baptême, la pénitence, un article du Credo ou d'une formule de prière, elle se présente sous la forme de courtes gloses liturgiques dans le cas du Rituel, reprises, dans le Catéchisme, et beaucoup plus élaborées sous forme de questions et de réponses, avec applications morales appropriées. Sortes d'avant-premières préparatoires à la célébration des fêtes mariales, ces gloses devaient être lues et commentées par les curés au prône de la messe paroissiale; par contre, les leçons du Catéchisme, préparées à l'intention des parents, visaient surtout l'instruction des enfants à domicile.

Les papiers officiels - et sous ce titre figurent les mandements, statuts, ordonnances, décrets synodaux, lettres patentes, correspondance officielle - sont des écrits d'ordre administratif ou disciplinaire. Leur contenu marial est forcément plus restreint, plus épars aussi mais remarquablement plus précis; il pénètre plus avant sur le terrain de l'engagement pratique et personnel et fait voir à quels gestes concrets l'évêque voulait entraîner la pratique mariale populaire. A titre d'exemples : chapelet en famille, évocation constante de la protection toujours efficace de Marie, dévotion à son Cour comme moyen efficace de raviver la ferveur chrétienne, appel à la responsabilité des curés pour cultiver chez leurs paroissiens une " solide dévotion "mariale ${ }^{4}$. Ici c'est le détail qui importe : il montre, par son choix, l'orientation personnelle de l'évêque et l'idée qu'il se faisait de la place et du rôle de Marie dans la vie concrète, quotidienne. Sans nul doute aussi que les pratiques encouragées correspondaient aux traditions du milieu ${ }^{5}$.

Les écrits didactiques voulaient apporter quelques éléments de doctrine, les écrits disciplinaires entendaient réglementer des attitudes; d'autres écrits à caractère plus personnel ajoutent à la pensée mariale de Mgr de Saint-Vallier une nouvelle dimension : la profondeur. Laissons de côté la correspondance privée; à cause de sa discrétion invincible, elle ne saurait enrichir notre inventaire. Par contre le sermon - un seul qui soit connu à date et peut-être le seul qui reste - est une pièce de valeur

4 Mandements des Evêques, I : 421.

Ibid. : p. 283. 
dans laquelle l'évêque explique, à un auditoire populaire, les exigences profondes d'une authentique dévotion mariale. Nous résumerons sa démonstration en disant qu'une dévotion mariale vraie doit déboucher sur un amour de conformité et s'intégrer aux structures essentielles de la vie chrétienne. Le titre condense bien le contenu du sermon :

discours sur l'obligation que nous avons d'imiter la ste Vierge en qualité de ses enfans par adoption qui avons este donnes à elle par Jésus christ mourant en croix en la personne de st Jean 6 .

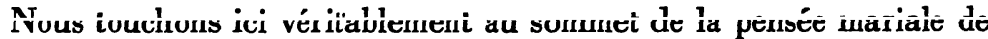
Mgr de Saint-Vallier. Il ne s'agit plus en effet seulement de pratiques mais d'un esprit qui colore toute la vie. La greffe qu'il propose s'exprime par un état de dépendance que n'auraient pas désavoué les tenants de l'Ecole Française. Une telle hardiesse de vision ne se retrouve nulle part ailleurs dans ses écrits. Il est bien plausible que ce discours soit avant tout l'écho d'un état d'âme propre au prédicateur.

Pour terminer cette exploration sommaire, on peut fixer quelques jalons provisoires.

Premièrement : quantitativement, le thème marial occupe une place, somme toute, plus que modeste dans les écrits de Mgr de Saint-Vallier. A ne considérer que les imprimés, la proportion globale est inférieure à une page contre 25; pour le reste on ne peut compter que de brèves allusions. Il n'y a donc pas lieu de parler de part exceptionnelle.

Deuxièmement: le modelé extérieur du thème est fort inégal. Cela tient au fait que l'évêque, à toute fin pratique, ne l'aborde jamais pour lui-même. Tantôt plus dense, tantôt plus incisive ou plus diluée, sa pensée mariale reste intimement liée, pour son développement, à certains cadres qui lui servent de support, telle en particulier la liturgie paroissiale, ou à quelque objectif précis comme, par exemple, la restauration d'un sanctuaire marial.

Troisièmement : le fait que cette pensée soit aussi dispersée, aussi étroitement conduite - hachurée devrait-on dire - par les circonstances, lui enlève beaucoup de relief.

C'est en prenant un peu de recul pour considérer la pensée dans son ensemble que l'on pourra plus aisément en apercevoir les traits dominants.

\section{II - Une pensée populaire, axée sur la liturgie}

L'action pastorale du successeur de Mgr de Laval prend place durant une période relativement tranquille : l'ère des expériences mystiques est close, l'élan missionnaire s'est ralenti, la vie religieuse se stabilise; c'est un état de repos qui contraste fort avec l'ardeur conquérante, si remarquable, de la période antérieure.

6 Le manuscrit se trouve aux archives de l'Hôpital Général de Québec. Différents Papiers : $\mathbf{n}^{\circ} 5$. 
Mais il n'y a pas que le contexte d'époque dont il faille tenir compte. Né, formé dans cet équilibre de l'esprit qui caractérise la seconde moitié du XVIIe siècle, Mgr de Saint-Vallier n'a pas, pour autant que l'on sache, vécu ces sortes d'engagements personnels exclusifs à promouvoir un privilège marial plus qu'un autre, comme ce fut le cas de Mgr de Laval et' des missionnaires jésuites pour l'ImmaculéeConception. Sa modération sur ce point le distingue déjà de ses devanciers, comme aussi le distingue sa docilité à suivre les pulsations spirituelles de son milieu d'accueil plutôt que de l'entraîner dans de nouveaux itinéraires.

Ces considérations expliquent jusqu'à un certain point la « monotonie» de la pensée mariale de Mgr de Saint-Vallier.

Par ailleurs le souci, toujours transparent chez lui, d'encadrer la dévotion populaire aura eu pour effet que sa pensée n'ait rien d'excessif dans ses tendances, rien de purement spéculatif dans ses procédés : elle est avant tout pratique, et par son orientation particulière, et par le choix des moyens ou des symboles utilisés pour soutenir le culte marial populaire. L'évêque fait constamment appel, pour ainsi dire, à l'expérience des gens. D'où les omissions de sa doctrine mariale. D'où encore son aspect volontaire et dépouillé de tout artifice. Quel que soit le mystère ou le privilège ou la vertu mariale qu'il développe, aucune échappée sentimentale ni effusion mystique, à la manière des écrivains spirituels du temps, ne vient rompre le rythme de l'exposé. Rien non plus qui fasse écho aux grands débats de l'époque sur les privilèges de Marie. L'évêque n'appartient à aucune école.

La conception qu'il a du rôle et de la place de Marie dans l'ascèse chrétienne populaire, plus suggérée que construite, trouve son unité et tout son poids dans l'importance singulière qu'il attache aux pratiques de dévotion. Son tempérament inquiet et ses préoccupations pastorales le prédisposaient à répondre de cette façon aux appels de la psychologie populaire. Ce qui frappe dans tous les exposés importants, c'est l'aspect refuge de la dévotion mariale qu'il enseigne. Certes il a soin de le motiver, en rappelant les titres qui fondent la puissance d'intercession de Marie : créature immaculée, Mère de Dieu sont de ceux qui reviennent le plus souvent. Leur mise en parallèle avec la misère et la faiblesse de l'homme leur donne encore plus de force. Il faut le reconnaître, la "protection efficace ", le "secours assuré ", les vocables "Mère de miséricorde », "avocate des pécheurs» sont des images hautement plus éloquentes pour la sensibilité populaire que les froids théorèmes d'une théologie mariale complète.

Cet appel constant à la sensibilité populaire constitue le caractère principal, foncier, de sa pensée mariale. Elle en a un autre, qui lui est complémentaire : c'est d'être une pensée moralisante. Mgr de SaintVallier ne s'arrête pas à considérer les vertus de la Vierge seulement pour en expliquer la beauté, c'est-à-dire pour elle-même. S'il rappelle son humilité, son obéissance, sa fidélité, sa pureté d'âme, c'est à la fin d'offrir, peut-on dire, un meilleur appât à la volonté du mieux vivre 
et une arme de combat. Comparée à la synthèse christologique de l'Ecole Française qui voulait qu'on honore la Vierge d'abord à cause de son excellence, la pensée mariale de Mgr de Saint-Vallier - par ce côté utilitaire - a quelque chose de négatif.

Que le pasteur invite à rendre culte à Marie pour en être aidé, à la regarder pour être mieux disposé, qu'il limite les exigences de la vie mariale aux pratiques de dévotion, tout cela n'est que le ressac d'un point de vue avant tout populaire. A la fin de son épiscopat cependant

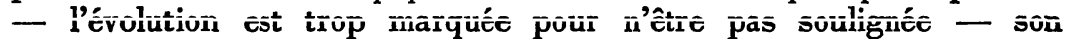
enseignement insiste davantage sur un engagement de vie. Si elle ne cesse pas d'être aussi intéressée, la dévotion mariale qu'il propose devient plus intérieure. Il ne lui suffit plus d'amener ses fidèles à s'appuyer avec confiance sur la bonté miséricordieuse de Marie; il indique, même avec une certaine âpreté, dans le directoire de la Confrérie du Cour de Marie $^{7}$ et dans son sermon, qu'un culte marial authentique ne saurait être satisfait de l'accomplissement de quelques gestes prescrits momentanés : le vrai dévot doit modeler ses dispositions intérieures sur celles de Marie. C'est une amorce discrète mais évidente d'une mise de vie à l'école de Marie.

Populaire par les horizons familiers et les thèmes concrets qui la caractérisent, la pensée mariale de Mgr de Saint-Vallier l'est aussi par les procédés mis en œuvre pour rejoindre plus sûrement l'audience du commun des gens. Sa façon de faire entrer le culte marial populaire dans le mouvement liturgique, de prendre appui sur l'action des parents pour prolonger celle des curés, nous apparaît comme un autre trait majeur de sa pensée mariale. La plus large part de son message, en effet, prend charnière sur les fêtes mariales célébrées au pays. L'évêque manifeste ainsi sa volonté d'axer la dévotion populaire sur la commémoration liturgique des privilèges et des mystères de Marie. Ce faisant, il a peut-être imité ce qui se pratiquait dans d'autres diocèses de France; le manque d'originalité n'infirme pas l'efficacité du moyen. Selon le principe des vases communicants, en l'occurrence la paroisse et la famille, il fait d'abord porter son effort et celui de ses prêtres sur la célébration paroissiale des fêtes mariales : c'est la raison d'être des gloses du Rituel. Les parents retrouvaient dans le Catéchisme, amplifiées mais dans le même ordre, la même doctrine et les mêmes applications. Grâce à ce retour continuel des mêmes vérités et des mêmes leçons pratiques, mises à commode portée, l'image de la créature privilégiée et toute-puissante s'enracinait dans les esprits, portant avec elle l'invite à la confiance.

Autant par les pratiques qu'elle suggère que par son orientation, la pensée mariale de Mgr de Saint-Vallier se raccordait, naturellement, à la tradition implantée dans la colonie depuis les origines. En plusieurs endroits de ses mandements, l'évêque fait appel à la coutume locale, notamment pour ce qui est du chapelet. On peut considérer comme un

7 Mandements des Evêques, I : 505-509. 
autre trait caractéristique de sa pensée qu'elle n'ait pas bouleversé les habitudes populaires déjà prises. Sous réserve toutefois qu'il n'a pas milité avec autant d'élan - nous l'avons dit - en faveur du culte spécial de l'Immaculée-Conception. Sous réserve encore qu'il s'est fait le propagandiste officiel du culte public rendu au Cour de Marie. Ces divergences mineures confèrent une note personnelle à tout ce que le contenu marial de ses écrits peut avoir d'impersonnel.

Utilitaire et moralisatrice dans son but, intégrée pour sa forme à la vie liturgique paroissiale, accessible par l'utilisation constante de l'imagerie populaire, traditionnelle en son ensemble, telles sont les caractéristiques de la pensée mariale de Mgr de Saint-Vallier. Prise en bloc, elle est de toute évidence le message direct d'un apôtre. Par les répétitions qu'elle contient et qui, à la simple lecture, peuvent la rendre monotone, par les soubresauts d'âme qu'elle manifeste, cette pensée porte une marque de vie que n'ont pas d'ordinaire les traités trop bien faits. Cette note de vie et le tour pédagogique qui en gouverne les manifestations sont, à mon sens, les traits les plus incisifs de la pensée mariale de Mgr de Saint-Vallier. Ils sont l'explication ultime de l'idée qu'il se faisait du rôle et de la place de Marie dans la vie chrétienne populaire.

Pour terminer, reconnaissons que Mgr de Saint-Vallier s'est montré, ici, plus conservateur qu'audacieux : il a suivi le courant, en l'éclairant, plus qu'il ne l'a précédé.

S'il faut chercher quelque originalité dans sa pensée mariale, ce n'est pas dans les sillons nouveaux qu'il aurait pu tracer, mais dans le fait qu'il a coordonné les éléments d'un culte populaire déjà en place; c'est d'avoir été le soutien autorisé de la tradition. En s'appuyant comme il l'a fait sur les cadres existants : famille, paroisse, liturgie, Mgr de Saint-Vallier a pu être un agent de stabilisation de la piété mariale en Nouvelle-France.

Par ses caractéristiques et par sa durée, ce message marial pourrait bien être considéré à la fois comme le témoignage d'un culte populaire et l'expression d'un idéal collectif d'une époque révolue. $A$ ces titres, il offre un point d'appui valable - croyons-nous - aux explorateurs du sentiment religieux des Canadiens des XVIIIe et XIXe siècles.

Hector BibEAU, s.m.m. Professeur d'histoire au Séminaire Montfort, Papineauville, P.Q. 